\title{
Effect of non-linearity in predicting doppler waveforms through a novel model
}

\author{
Aman Gayasen*1, Sunil Kumar Dua ${ }^{1}$, Amit Sengupta² and D Nagchoudhuri ${ }^{3}$
}

Address: ${ }^{1}$ Department of Electrical Engineering, Indian Institute of Technology, New Delhi, India, ${ }^{2}$ Central Health services, Specialist Polyclinic (CGHS, Mumbai) India and ${ }^{3}$ Department of Electrical Engineering, Indian Institute of Technology, New Delhi. India

Email: Aman Gayasen* - gayasen@cse.psu.edu; Sunil Kumar Dua - sunil.dua@mailcity.com; Amit Sengupta - senguptaamit@hotmail.com; D Nagchoudhuri - dnag@ee.iitd.ernet.in

* Corresponding author

Published: 18 September 2003

BioMedical Engineering OnLine 2003, 2:16
Received: 10 July 2003

Accepted: 18 September 2003

This article is available from: http://www.biomedical-engineering-online.com/content/2/1/16

(C) 2003 Gayasen et al; licensee BioMed Central Ltd. This is an Open Access article: verbatim copying and redistribution of this article are permitted in all media for any purpose, provided this notice is preserved along with the article's original URL.

\begin{abstract}
Background: In pregnancy, the uteroplacental vascular system develops de novo locally in utero and a systemic haemodynamic \& bio-rheological alteration accompany it. Any abnormality in the non-linear vascular system is believed to trigger the onset of serious morbid conditions like preeclampsia and/or intrauterine growth restriction (IUGR). Exact Aetiopathogenesis is unknown. Advancement in the field of non-invasive doppler image analysis and simulation incorporating nonlinearities may unfold the complexities associated with the inaccessible uteroplacental vessels. Earlier modeling approaches approximate it as a linear system.

Method: We proposed a novel electrical model for the uteroplacental system that uses MOSFETs as non-linear elements in place of traditional linear transmission line (TL) model. The model to simulate doppler FVW's was designed by including the inputs from our non-linear mathematical model. While using the MOSFETs as voltage-controlled switches, a fair degree of controlled-nonlinearity has been introduced in the model. Comparative analysis was done between the simulated data and the actual doppler FVW's waveforms.

Results \& Discussion: Normal pregnancy has been successfully modeled and the doppler output waveforms are simulated for different gestation time using the model. It is observed that the dicrotic notch disappears and the $S / D$ ratio decreases as the pregnancy matures. Both these results are established clinical facts. Effects of blood density, viscosity and the arterial wall elasticity on the blood flow velocity profile were also studied. Spectral analysis on the output of the model (blood flow velocity) indicated that the Total Harmonic Distortion (THD) falls during the mid-gestation.

Conclusion: Total harmonic distortion (THD) is found to be informative in determining the Fetomaternal health. Effects of the blood density, the viscosity and the elasticity changes on the blood FVW are simulated. Future works are expected to concentrate mainly on improving the load with respect to varying non-linear parameters in the model. Heart rate variability, which accounts for the vascular tone, should also be included. We also expect the model to initiate extensive clinical or experimental studies in the near future.
\end{abstract}




\section{Background}

The biological system is highly non-linear. Therefore, the output of any simulated model will be near actual only when it includes various non-linearities. In such a case, unlike a linear system, minor variations in input may result in absolutely unpredictable outputs. In pregnancy, the local geometric vascular alteration is also accompanied by the systemic bio-rheological and haemodynamic change. Any abnormality in the system is known to trigger the onset of serious morbid conditions like pre-eclampsia and/or intrauterine growth restriction (IUGR). They are one of the leading causes of maternal and fetal deaths in both the developing as well as the developed countries. Precise Aetiopathogenesis is still not clear but the disorder has been linked to the endothelial dysfunction and the uteroplacental insufficiency, which in turn triggers systemic hypertension and sympathetic overactivity [1-3]. Studies by the standard biomedical technique have failed to improve our understanding of the rapidly altering uteroplacental blood flow dynamics during pregnancy (Fig 1). Advancement in the field of non-invasive Doppler image analysis and simulation incorporating non-linearities may unfold the complexities associated with the inaccessible uteroplacental vessels. Modeling is a known tool in the physiological fluid dynamics study. A validated nonlinear mathematical model of the uteroplacental system has been developed which has also opened up a new frontier of research in the field of Doppler velocimetry analysis, modelling and image processing [4]. An electrical model can be easily simulated which can also incorporate various non-linearities present in the actual system. Earlier, Doppler FVW's have been analyzed by computer based algorithms using simple linearized first order differential equations transforming the functions into an $\mathrm{R}-\mathrm{C}$ circuit commonly used in haemodynamic investigation [5]. Such a Transmission Line (TL) model [6] is linear, whereas, the actual system is non-linear and active. Blood flow velocity profile at the distal uteroplacental vessels (UPV) contains extra frequencies compared to the proximal uterine artery (UA). To analyze the FVW's mapped at the level of uteroplacental vessels, one has to separate it from the uterine artery, and it needs manipulation to see the effect of UPV on UA and vice-versa. Thus we need an active device that represents pregnancy time and change, which is not possible with TL model. Our group made an attempt to device a theoretical Junction field effect transistor (JFET) model earlier $([7,8]$. MOSFET, field effect transistor, widely used in electrical engineering is flexible, and is nearer to the non-linear biological system. While using the MOSFETs as voltage-controlled switches, a fair degree of controlled-non-linearity has been introduced in the model. This is a distinct advancement and a major gain, more so in pregnancy, over the previously described passive linear models. In addition to the effect of the vessel elasticity and the blood density, the effect of blood viscos- ity is also simulated. Moreover, wide availability of several MOS models and extensive research in MOS fabrication justifies its selection as an ideal and a better model than the TL model. The simulation outputs are similar to the actual Doppler FVW's profiles obtained for some of the non-linear parameters by various authors [4,9]. However, we expect the present model and the simulation results to initiate extensive clinical or experimental studies involving various non-linear parameters in the near future. The feedback will help the scientist working in the field of modelling to further modify and improve the model significantly.

\section{Method \& Model Formulation \\ The anatomy and pathophysiology of the utero-placental system}

For the maintenance of the normal pregnancy, the placenta, a fetomaternal organ, plays an important role. It is developed by the interaction of the maternal spiral end vessels and the trophoblastic villi. The spiral end vessels are the branches of the radial artery, which comes out from the arcuate arteries at right angles from the main uterine artery. Brosen (1967) proposed that for normal fetoplacental flow, the growing trophoblasts invade the spiral vessels during formative stages beyond the deciduomyometrial junction converting the entire system into a low-resistance shunt. The spiral vessels lose the normal architecture and the elasticity and become a low resistance, dilated, straight, vein like vessel. The diameter increases from $0.5 \mathrm{~mm}$ to $1.0 \mathrm{~mm}$. The main proximal uterine artery, supplying most of the blood to the system, also gets dilated with a relative fall in the resistance, and the diameter increases from $2.0 \mathrm{~mm}$ to approximately 5.0 $\mathrm{mm}$. In total, 120 such vessels are affected, discharging the blood into intervillous space and displacing the villi with the maternal arterial pressure head. The capacitance increases to allow the excess demand of blood flow during pregnancy. In abnormal condition such as Intra-Uterine Growth Restriction (IUGR) and pre-eclampsia (PE), the increase in diameter has not been observed. The detailed architectural geometric patterns are given in Fig. 1 .

\section{The electrical model}

The model is based upon the transmission line model described in [6]. We proposed the mathematical model for uteroplacental artery, which contributes to the load at the end of the transmission line [4]. Various inputs used in the model representing the non-linear parameters such as elastic parameters, viscosity, wall density, womersley parameters are taken from the known experimental and histopathological studies and are further modified for specific conditions by using standard formula [4]. Based on the accumulated knowledge, the entire system has been simplified to develop a novel MOSFET model. MOSFET is the ideal choice for this model, because (i) it 


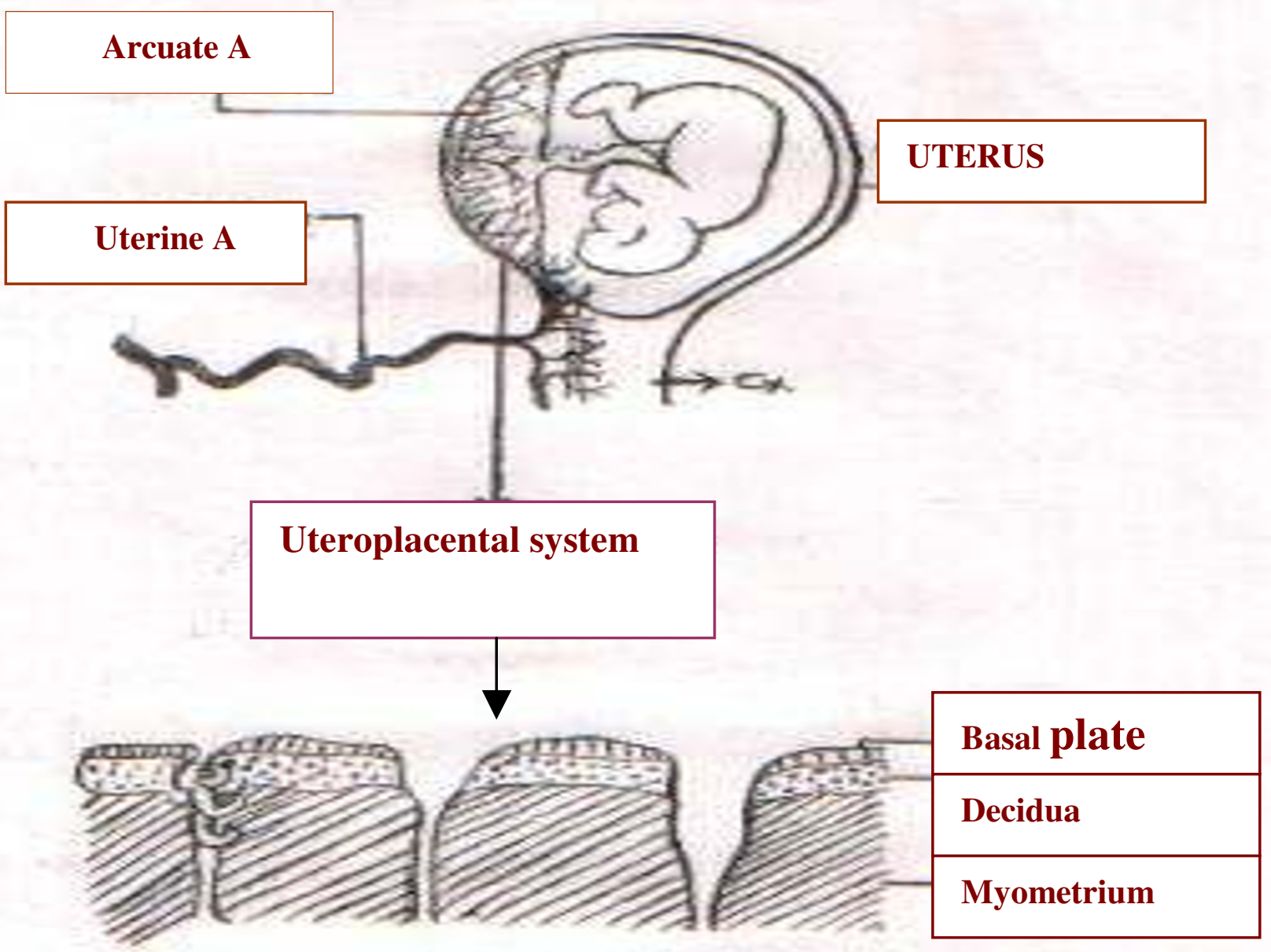

Figure I

Diagrammatic representation of the conversion of the spiral arteries into uteroplacental vessels

exhibits the desired non-linear current-voltage characteristics, (ii) simulation models of MOSFET are easily available and (iii) MOSFETs are easy to fabricate with state of the art technology. A schematic representation of the proposed model is shown in Fig. 2. All circuit elements in the transmission line model that vary with pregnancy time are replaced by some kind of MOSFET-based active structure (Appendix). These elements are shown as boxes in Fig. 2. The model consists of the following components.

1. Current Source: A current source in the model represents the blood flow input to the biological system. It 


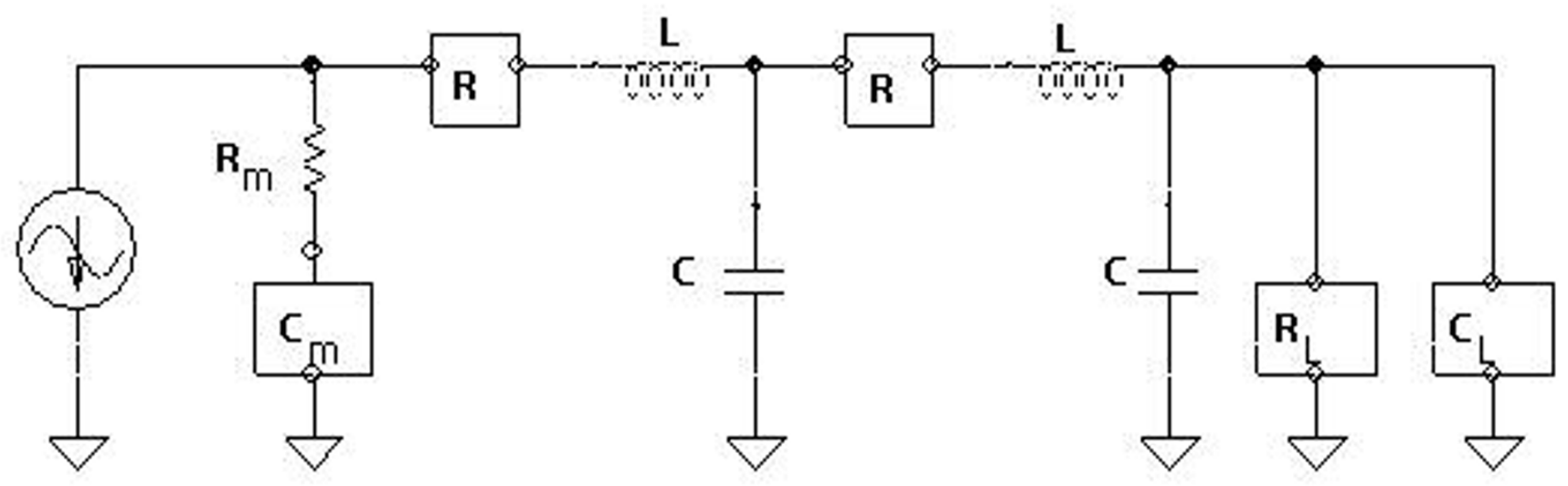

Figure 2

Proposed MOSFET model

consists of 4 frequencies, which we have taken from the TL model. The actual implementation was done using 4 sinusoidal current sources connected in parallel.

2. Matching Impedance $\left(\mathrm{R}_{\mathrm{m}}, \mathrm{C}_{\mathrm{m}}\right)$ : The matching impedance is needed to absorb the waves that come after reflection from the load.

3. Uterine Artery: As in case of the TL model, the uterine artery is represented by an RLC ladder-type structure. The difference is the implementation of resistance (MOSFETs are used to implement resistance, to give more flexibility), and also the inductance, which is made constant. Implementation details are discussed in Appendix.

4. Load: The uteroplacental system is lumped with the intervillous sinus and approximated by a capacitive bank in parallel with a MOSFET resistance (Appendix).

The uteroplacental system is considered in isolation from the rest of the circulatory system. External influences on the system have not been considered.

\section{Modification over transmission line model}

The architecture of the proposed model is based on the TL model. It has been modified in the following ways.

The proposed model simulates the uteroplacental system at any given pregnancy time. Pregnancy time is an input to the model, which internally affects the value of a voltage source, (which in turn change the circuit parameters). This must not be confused with the real time, and the sys- tem remains time invariant. The system can be simulated for an arbitrary pregnancy time by changing the "pregnancy time" input accordingly.

In the TL model, the uterine artery is modeled as a transmission line with the uteroplacental portion forming its load, which is also linear, consisting of a resistance and a capacitance. In the proposed model, it is assumed that as pregnancy matures, load of the TL model changes nonlinearly.

The elements of transmission line are made to vary with respect to a voltage, which is controlled by the input "pregnancy time". These elements have been shown in boxes in Fig. 2. MOSFETs have been used to implement such voltage-controlled parameters.

In the TL model, inductance of the vessel also varies with pregnancy time. It is difficult to implement a voltage-controlled inductance using MOSFETs [10]. Therefore, we removed this variation in inductance through some mathematical manipulations (Appendix), thereby, making both, inductance and capacitance of the vessel constant with pregnancy time. The variation in them is effectively mapped onto the load of the transmission line. Thus, now the load of the transmission line does not represent only the uteroplacental arteries and the intervillous sinus. Rather, some variations in the uterine artery also affect the load. Modeling of the load has been improved. In our model, load capacitance decreases as pregnancy matures. This voltage varying capacitor has been implemented using a capacitor bank. MOSFET switches control the 
actual number of capacitors that add in parallel in the circuit. This is the major non-linear element in the model and all the non-linearities like elasticity, viscosity, density are studied using the equations governing the capacitors (Appendix).

\section{Actual doppler flow velocity waveform analysis}

The video recording of Doppler FVW's measured at the level of the uterine or the arcuate artery were digitized and the frames were taken out using a tool available with the special workstation provided by the silicon graphics (SGI). The application is known as "Camera" (Fig. 3). To analyze the FVW, an envelope was extracted for one period and around 75 samples were taken from it. Software was developed in $\mathrm{C}++$ to approximately determine the magnitude of Fourier Series coefficients. We mapped all the periods on a fixed time of 0.83 seconds, which is a normal heart beat period and took frequency domain average of all such periods. We considered FVW's of equal time frame for purpose of analysis, in order to remove the time variations, which is to the order of $\pm 10 \%$ analyzed for a single patient. The problem was tackled by mapping all the periods on a fixed time of 0.83 seconds, which is a normal standard heart beat period. We took frequency domain average of all such periods analyzed for a single patient. Three patients representing three different conditions and gestation period were analyzed and the following results obtained. In abnormal pre-Eclamptic condition (patient 3), lower harmonic magnitude is lesser compared to the normal pregnancy (Fig. 4). The actual Doppler FVW's used for the analysis are collected from the records of the mothers undergoing routine diagnostic Doppler and sonography tests in our hospital clinic for the various pregnancy disorders as well as the established data from the published literature. The analysis was done primarily to validate the model, secondly to understand the total harmonic distortion and thirdly to propose different Doppler FVW's pattern that may emerge for various non-linear inputs which can be used to initiate further comparative and experimental studies in future.

\section{Results and Discussion}

The documentation of the Doppler flow-velocity waveforms from the key peripheral uteroplacental vessels, the main functional site, is necessary to ascertain the underlying haemodynamic changes in pregnancy. The failure to obtain the information due to technological limitations as well as complex geometric pattern and variation existing in the system has been a major impediment to the advancement in knowledge $[4,7,11]$. Computer based algorithms and electrical transmission line analogy using mathematical model has been reported [5]. Simulations are done taking into consideration functions like lumped proximal and distal resistance, mean arterial pressure without explaining the geometric and the flow condi- tions. A definite pressure-flow velocity relation of the uteroplacental system considering its morphological and rheological changes, an important physiological parameter in pregnancy has not been considered as yet. In the absence of actual recording an alternative useful rational approach has been tried here linking the accessible proximal vessel to the unknown distal system using a theoretical MOSFET model. Normal pregnancy has been successfully modeled using the model. Output waveforms for increasing pregnancy time are shown in Fig. 5. It is observed that dicrotic notch disappears as pregnancy matures. Also, S/D ratio decreases with pregnancy time. Both these results are established clinical facts. As the pregnancy progresses, there is a gradual fall in the uteroplacental vascular resistance with increasing capacitance to accommodate extra blood volume necessary for the fetomaternal perfusion and exchanges. The Actual Doppler velocity profile recorded at the level of the uterine artery in the normally progressing pregnancy also shows a gradual fall in the Pulsatility and the resistance index $[4,12,13]$. In the present study, other than validating the non-linear electrical model, spectral analysis on these outputs showed some interesting results. Magnitudes of Fourier Coefficients of the outputs are plotted in Fig. 6. It is observed that the first harmonic is larger than fundamental frequency in early pregnancy. As pregnancy matures, the fundamental frequency component increases sharply and then shows somewhat linear fall as frequency (harmonics) increases. Similar relation is seen in hypertensive condition, where systolic (S) peak is higher and diastolic (D) is low (High S/D ratio), but the rise is comparatively and correspondingly lower compared to normal pregnancy. This result has been earlier observed using spectral analysis on actual Doppler data [14]. Also, Total Harmonic Distortion (THD) of the outputs is plotted vs. pregnancy time in Fig. 7. It is observed that the THD decreases as pregnancy matures. This too is expected, as it is known that low frequency components increase with pregnancy time [14]. Therefore, not only, we could generate similar type of variations in the FVW's profile as seen in the actual pregnancy condition, but also simultaneously, we found THD as an important diagnostic parameter that may be considered in future. A FVW's from mid pregnancy with high THD signifies high uteroplacental resistance and thus may predict impending pre-eclampsia and /or IUGR. The very fact that the results obtained from the mathematical model, the real time Doppler \& spectral analysis and the non-linear electrical MOSFET simulation are in close approximation proves that the model is a novel one. The correspondingly similar simulated output that we obtained through this non-linear model by manipulating the terminal load and introducing the extra frequencies, the vital non-linear factor which greatly effects both the maternal and fetal haemodynamic is the biggest gain over the linear model. 


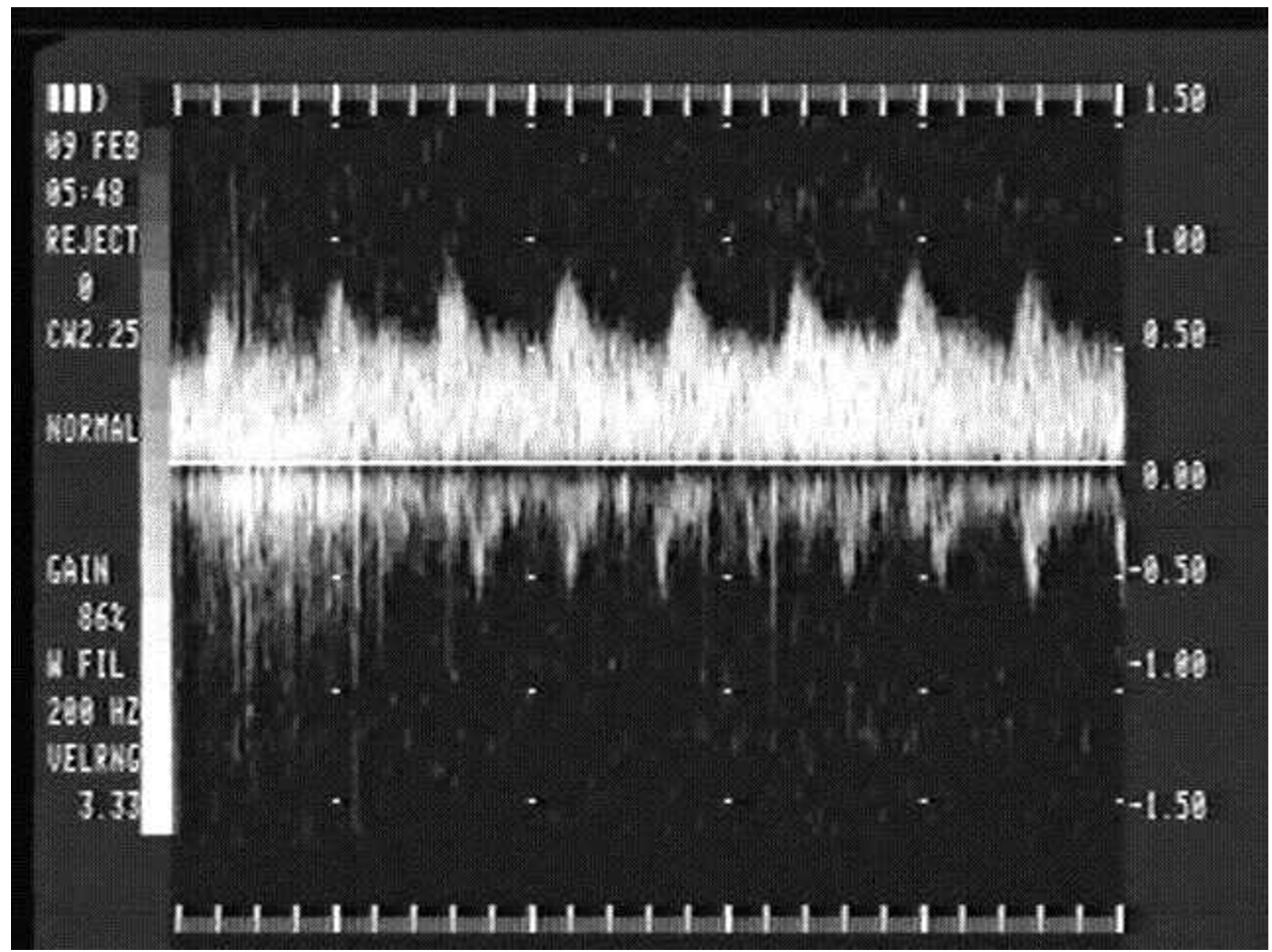

Figure 3

A frame extracted from doppler FVW

\section{Density of the blood and the flow velocity profile}

The effect of the blood density on the blood flow velocity has been observed using the model. The results, for density variation from 0.2 to 9.3 times the normal for 24week pregnancy are plotted in Fig. 8. As density is decreased, the waveform becomes flatter. These observations indicate that blood FVW acquires more features of a normal pregnancy as density decreases. It is a well-known fact that haemodilution occurs in normal pregnancy; thus, the decrease in blood density may be one of the causes for the features in FVW that are normally considered to denote normal pregnancy. It is also known that the resistance to flow varies with density, more so at low shear rate. As the density increases due to increase in fibrinogen level, globulin, RBC agglutination, decrease in RBC filterability in plasma, the haematocrit increases and so does the cardiac output and resistance. Similar relation may not exist at high shear rates $[15,16]$. It is observed that systolic peak increases as density is increased. Therefore, we could successfully simulate the actual patho-physiological condition using this non-linear model for the first time. Such phenomenon justifiably explains high resistance flow in Pre-eclamptics. This has been a major advancement in electrical modeling and Doppler waveform analysis. However, its effect on diastolic waveform needs some further analysis. The fall in the THD is acute and sharp when the density increases many folds theoretically. Such rise in density does not exist in the real biological system. However, a minimal change in THD is seen when the density is within the biological range. A definite explanation to the THD variation may depend 


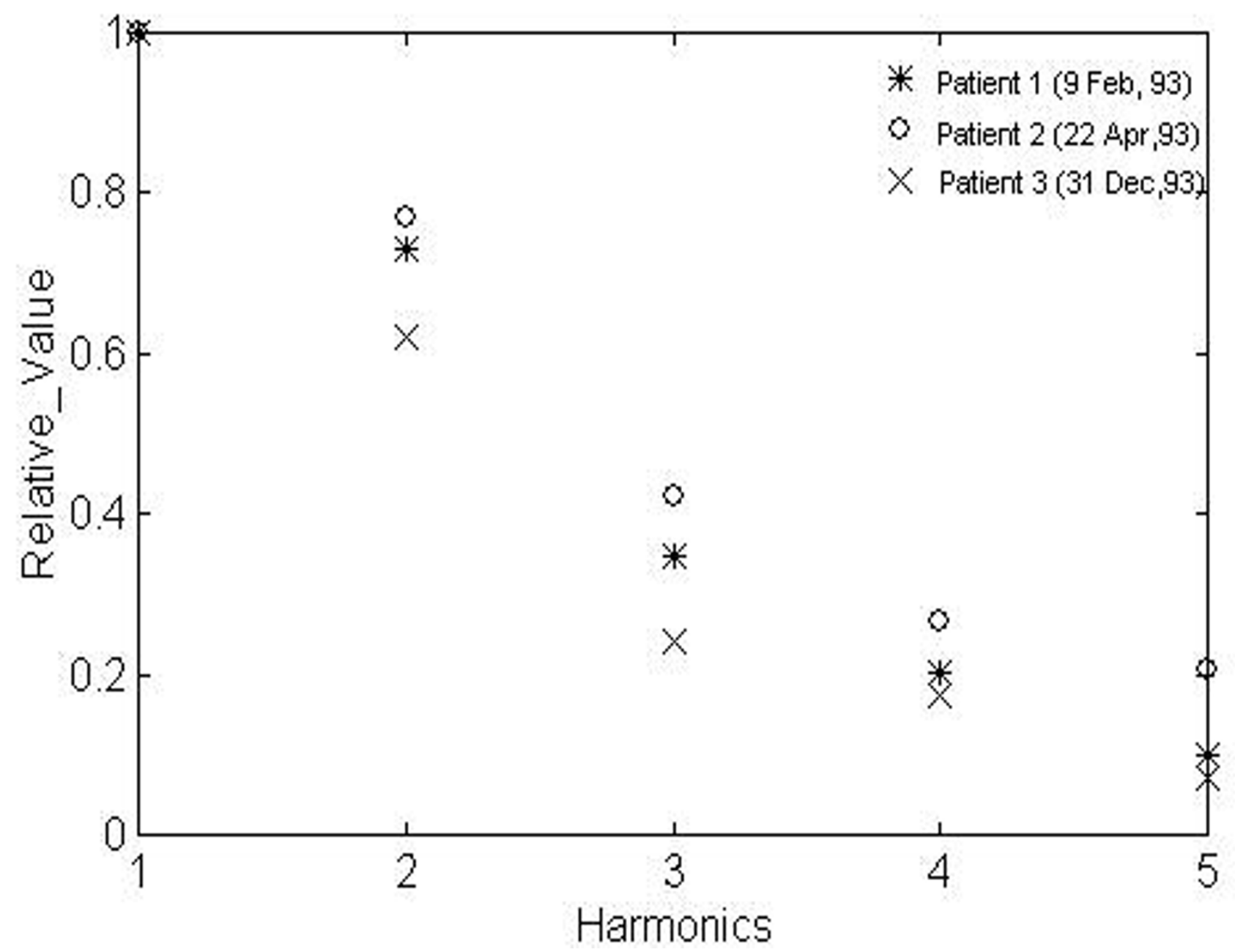

Figure 4

Frequency content of doppler data for three patients

upon many other interrelated variations such as shear rate, elasticity, viscosity, radius etc.

\section{Vessel elasticity and the flow velocity profile}

Effect of arterial wall elasticity on blood FVW is shown in Fig. 9. The Young's modulus of elasticity is varied from 0.1 to 10 times the normal value for normal 24-week pregnancy. For very low elasticity or a flaccid condition, low systolic and high diastolic peaks are observed. This effect has been observed clinically. Also, increasing elasticity beyond around twice the normal had negligible effect on the waveform. Thus elasticity in these smaller vessels may not be an important factor in determining the flow profile or flow conditions or reason thereof for underlying normal or abnormal pregnancy related flow changes or pathology as suggested by Brosen [1]. Similar conclusion has also been drawn in our previous work on mathematical model [4]. But, there may be other important factors such as rheological parameters, geometry, overall cardiac 


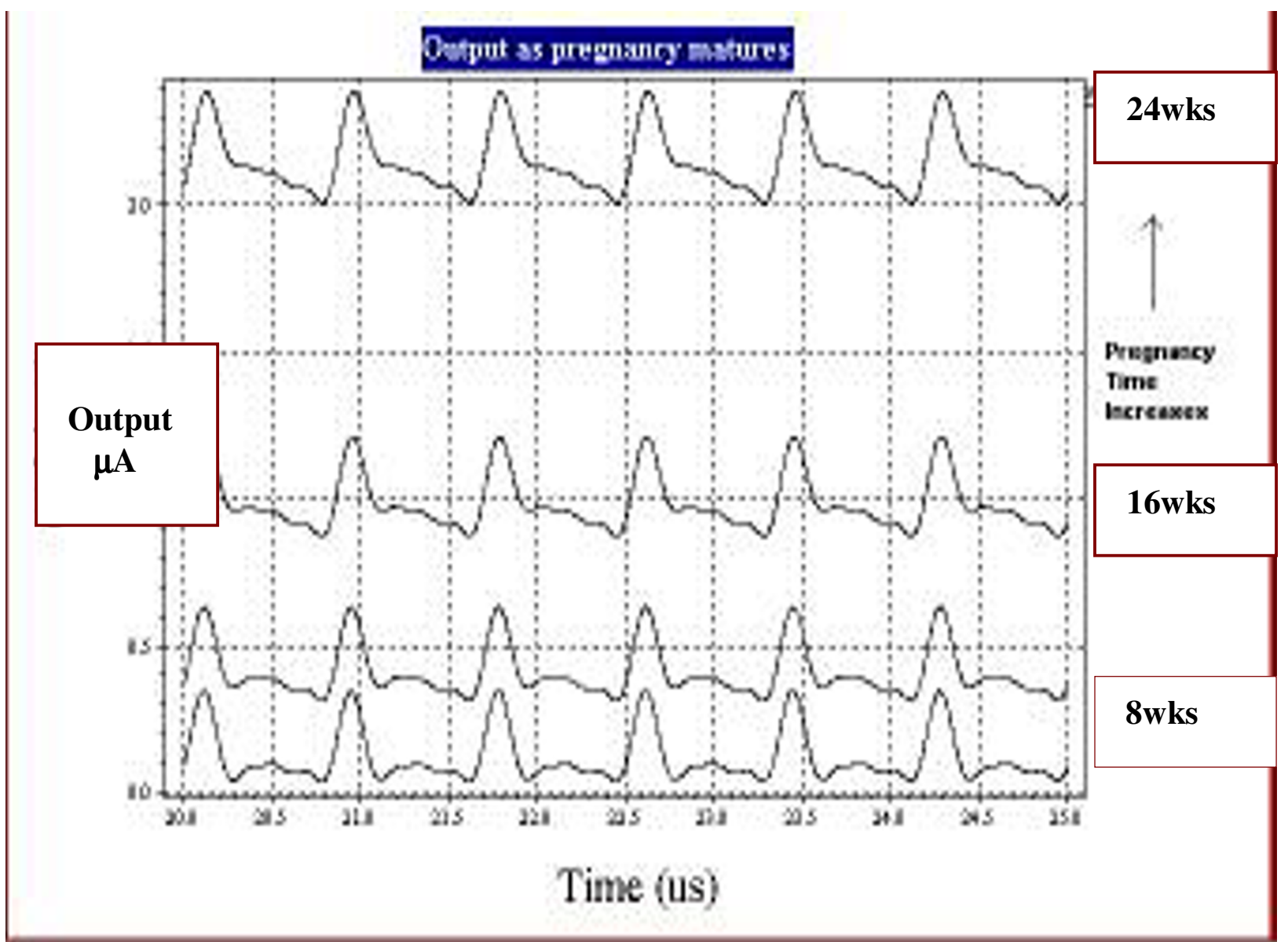

Figure 5

Normal outputs for increasing pregnancy time

output (blood volume), heart rate variability, and overall load at the distal end or proximal area, playing a role.

\section{Blood viscosity and the flow velocity profile}

Variation in blood FVW with viscosity appears to be negligible. We varied the viscosity from 0.1 to 10 times the normal value. On decreasing viscosity from normal, waveforms are indistinguishable from each other. On increasing the viscosity, though, there is a small decrease in the systolic peaks. Viscosity per se may not effect the flow velocity waveforms in arteries as inertial forces predomi- nate. Only in microvessels does it predominate over inertial forces. Viscosity has been shown not to influence the Doppler flow profile in pregnancy [9].

\section{Conclusions}

Advancement in the field of non-invasive Doppler image analysis and simulation incorporating non-linearities may unfold the complexities associated with the inaccessible uteroplacental vessels. An abnormality in the non-linear vascular system is known to trigger a serious pathological condition of pre-eclampsia and/or 


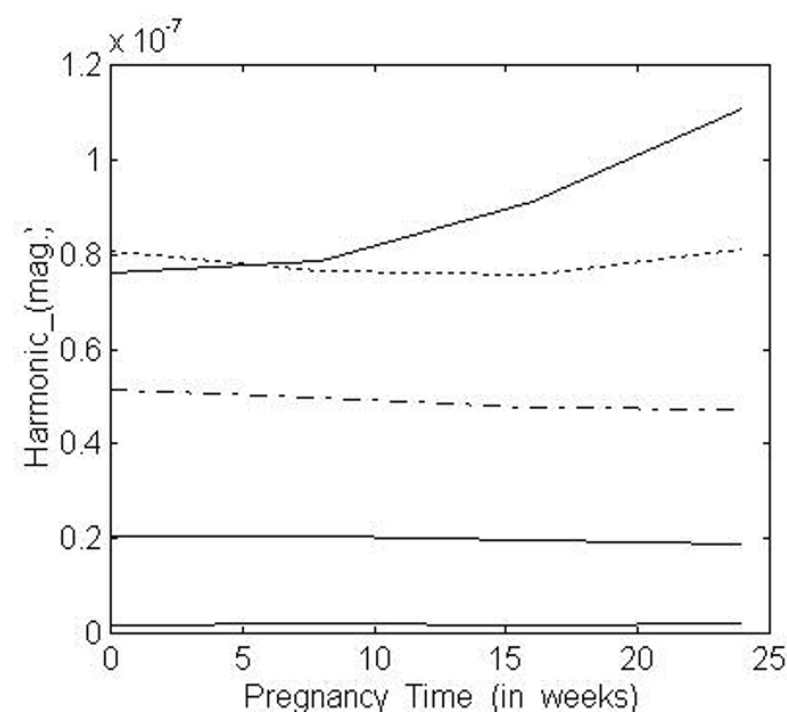

Figure 6

Harmonics vs. pregnancy time

intrauterine growth restriction (IUGR); one of the leading causes of Feto-maternal deaths the world over. A non-linear mathematical model of the uteroplacental system has already been developed and validated using the available Doppler FVW's studies only to a certain extent [4]. As a further advancement in modeling, a non-linear MOSFET electrical model using the load as derived by the mathematical model for the utero-placental system has been proposed in the present study, which gives expected results and matching FVW's outputs for the normal as well as the abnormal pregnancy. Effects of the blood density, the viscosity and the elasticity changes on the Doppler FVW's profile are simulated. Total harmonic distortion (THD) is found to be informative and valuable in determining the Feto-maternal health. The Future works is expected to concentrate mainly on improving the load with respect to varying non-linear parameters in the model. These shapes may be incorporated in the model. Also, the heart rate has been assumed to be constant in our model. But in actual system, the heart rate varies with the time and is known to play an important role in determining the underlying cardiovascular sympathetic activity. The heart rate variability should also be included in the future model.

\section{Appendix}

Replacing a time varying inductor with constant inductor: In the transmission line model, transmission line parameters vary as derived below $[6,18]$-------- (1).

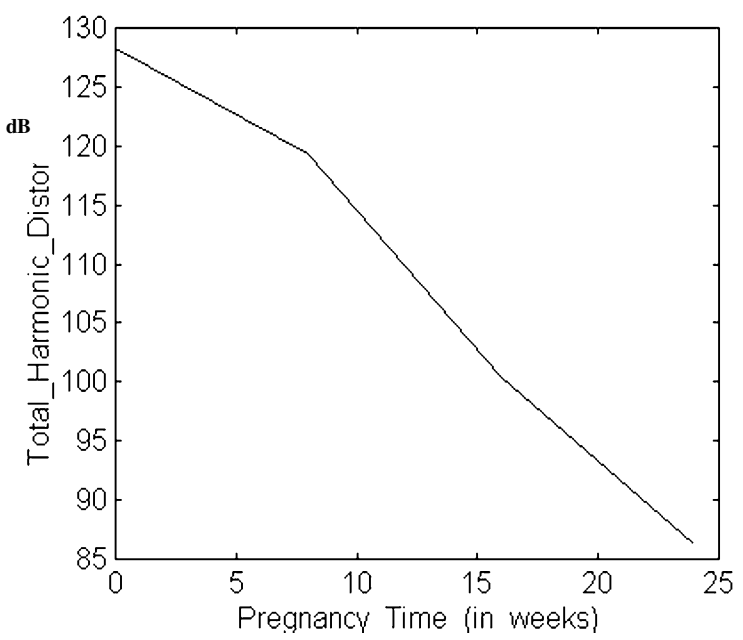

Figure 7

THD vs. pregnancy time

$R=\frac{81 \eta}{8 \pi r_{0}^{4}}$ (in ohms/unit length); $L=\frac{9 \rho}{4 \pi r_{0}^{2}}$ (in Henrys/unit length)

$C=\frac{3 \pi r_{0}^{3}}{2 E h}$ (in farads/unit length)

Where $r_{0}$ is the vessel radius, $E$ is the Young's modulus of the vessel wall, $h$ is the vessels wall thickness, $\rho$ is the mass density of blood, and $\mathrm{H}$ is the blood viscosity. We notice that the transmission line model has an inductive element that varies with pregnancy time. It is difficult to make an inductance using active elements [10]. So, we removed this variation in inductance by mapping the change in inductance to other circuit elements. This was achieved through some mathematical manipulations, as explained below.

The transfer function for RLC-circuit implementation of transmission line model is:

$\frac{I_{\text {out }}}{I_{\text {in }}}=\frac{1}{\left\{1+s C R+s^{2} L C+\frac{s C R_{l}}{1+s\left(C+C_{l}\right) R_{l}}\right\}\left\{1+\frac{s C R_{l}}{1+s C_{l} R_{l}}\right\}}$

where $I_{\text {out }}$ is the output current; $I_{\text {in }}$ is the input current; $R$, $C$ and $L$ are transmission line resistance, capacitance and inductance respectively; and $R_{l}$ and $C_{l}$ are load resistance and capacitance respectively. In order to make the inductance of transmission line independent of pregnancy time, we make the following transformations in the circuit. 


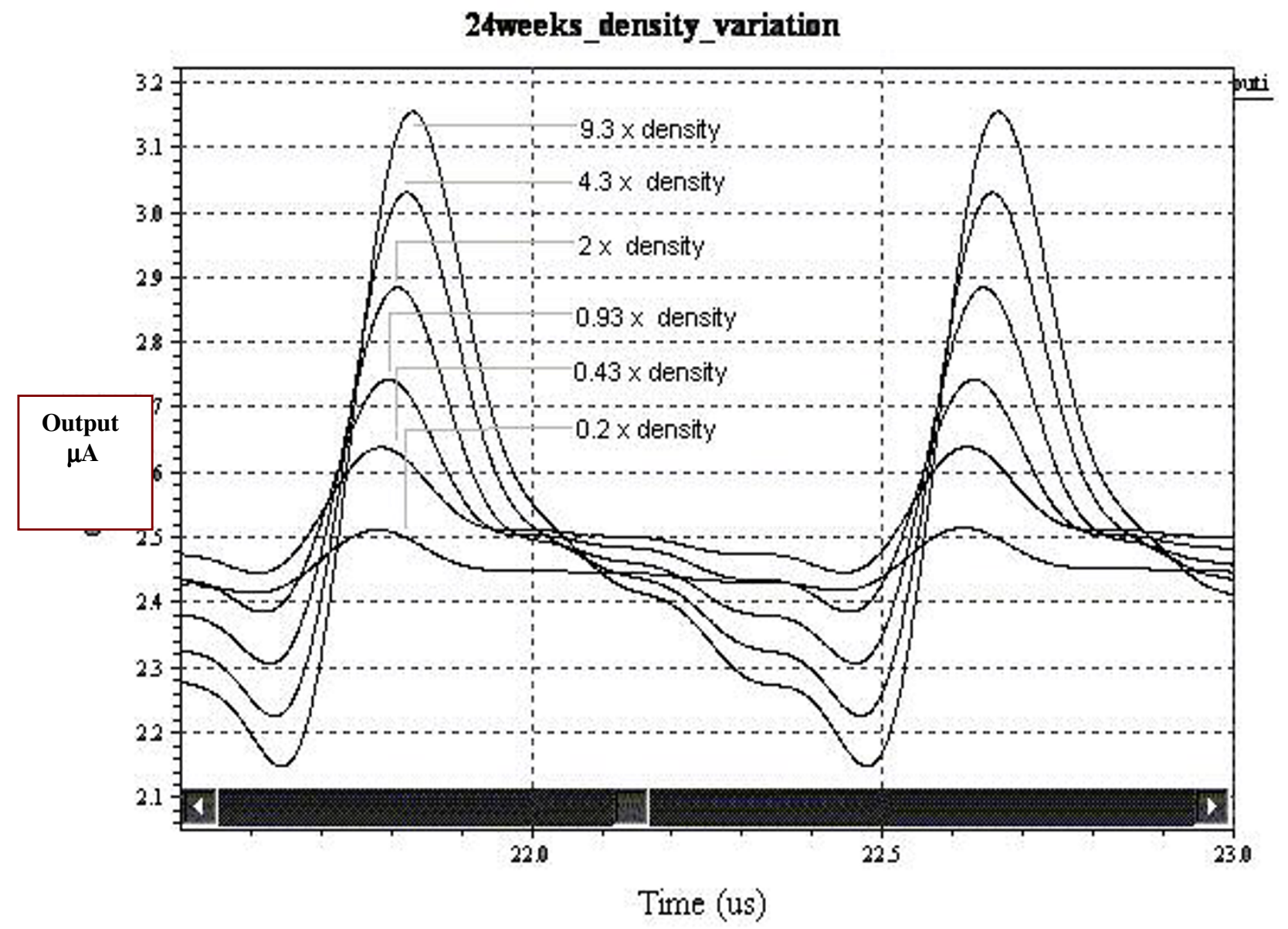

Figure 8

Density variation

$L \rightarrow 1 ; C \rightarrow L C ; C_{l} \rightarrow L C_{l} ; R \rightarrow R / L ; R_{l} \rightarrow R_{l} / L$

Note that this does not alter the transfer function of the TL model. Thus, equations for the transformed circuit elements become:

$$
\begin{aligned}
& R^{\prime}=\frac{R}{L}=\frac{9 \eta}{2 \rho r_{0}^{2}} \\
& C^{\prime}=L C=\frac{27 \rho r_{0}}{8 E h}
\end{aligned}
$$

As, $h=0.1 r_{0}$ for normal pregnancy, the capacitance becomes independent of $r_{0}$. This enables us to use a passive capacitor to represent the transmission line capacitance. It may be observed that with this transformation, the transfer function is effectively represented in terms of time constants of the circuit.

This transformation resulted in an equivalent model for the micro-vascular system. But the disadvantage of this exercise is that the model is no more directly analogous to the biological system. For example, in the modified model, the load capacitance is a function of radius of the vessel as well as the mass density of blood. This has no direct analogy in the biological system. Also, there is no longer a direct analogy between pressure in biological system and voltage in the model. But the resulting simplification in implementation justifies this transformation. Thus, in the modified model, inductance remains constant throughout the pregnancy period. The value of inductance is kept at $10 \mathrm{uH}$. 


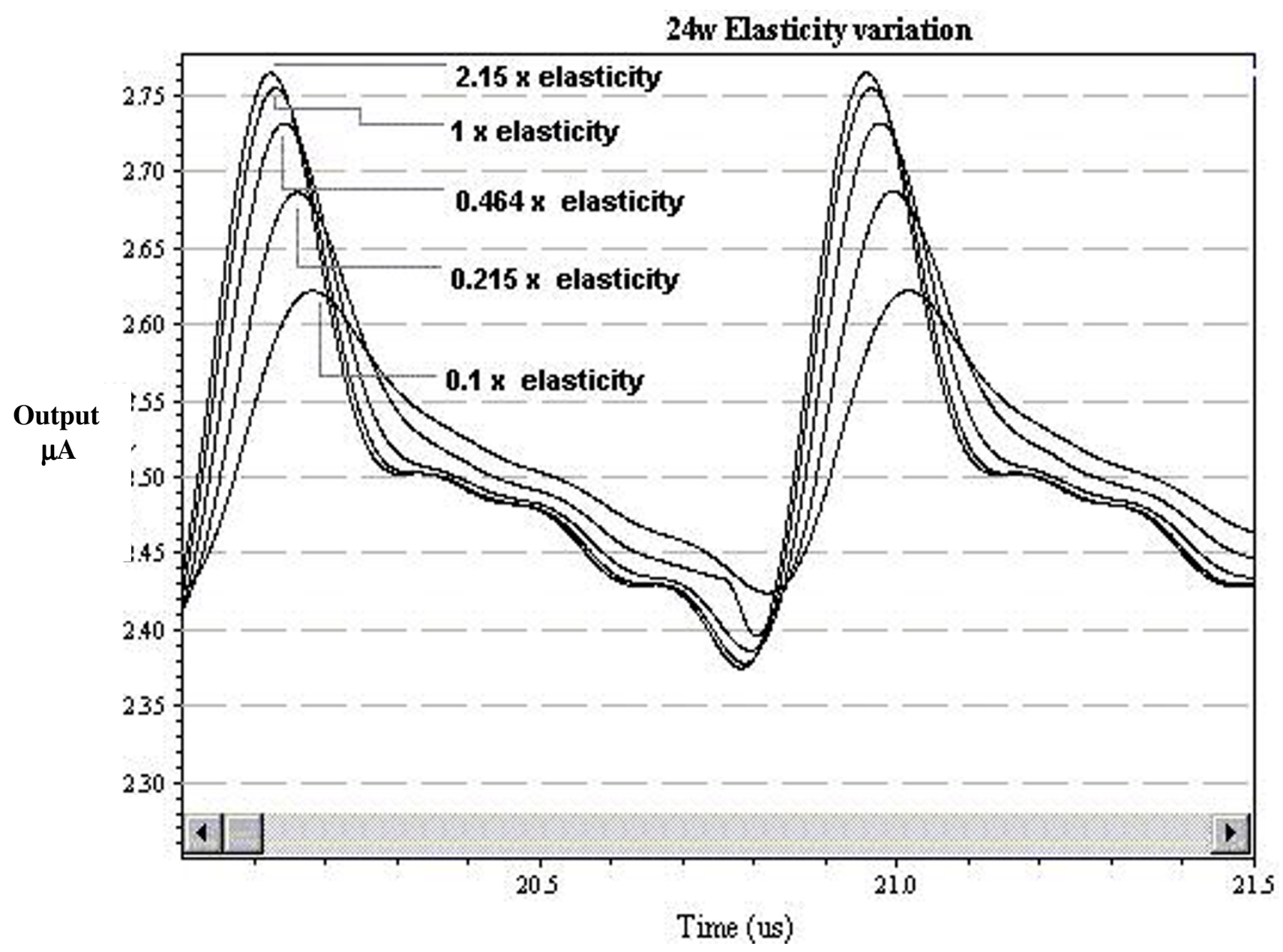

Figure 9

Elasticity variation

\section{Implementation of circuit elements}

Transmission line resistance

The transmission line resistance is implemented using channel resistance of an NMOS. Channel resistance has the following expression [17], when the NMOS is in triode region.

$$
r_{d s}=\frac{1}{k\left(\frac{w}{L}\right)\left(V_{G S}-V_{T}-v_{d s}\right)}
$$

Where $k$ is gain factor of NMOS, $W$ is width of channel, $L$ is length of channel, $V_{G S}$ is gate voltage, $v_{d s}$ is drain voltage, $V_{T}$ is threshold voltage of MOSFET.
Assuming low $v_{d s^{\prime}}$

Thus, if the gate voltage were made to vary proportional to pregnancy time,

$r_{d s}=\frac{1}{k\left(\frac{w}{L}\right)\left(V_{G S}-V_{T}\right)}$

the resistance of uterine artery would decrease as pregnancy matures. This is as expected, in normal pregnancy. Assuming a linear variation of radius in equation (3) with pregnancy time, we get 


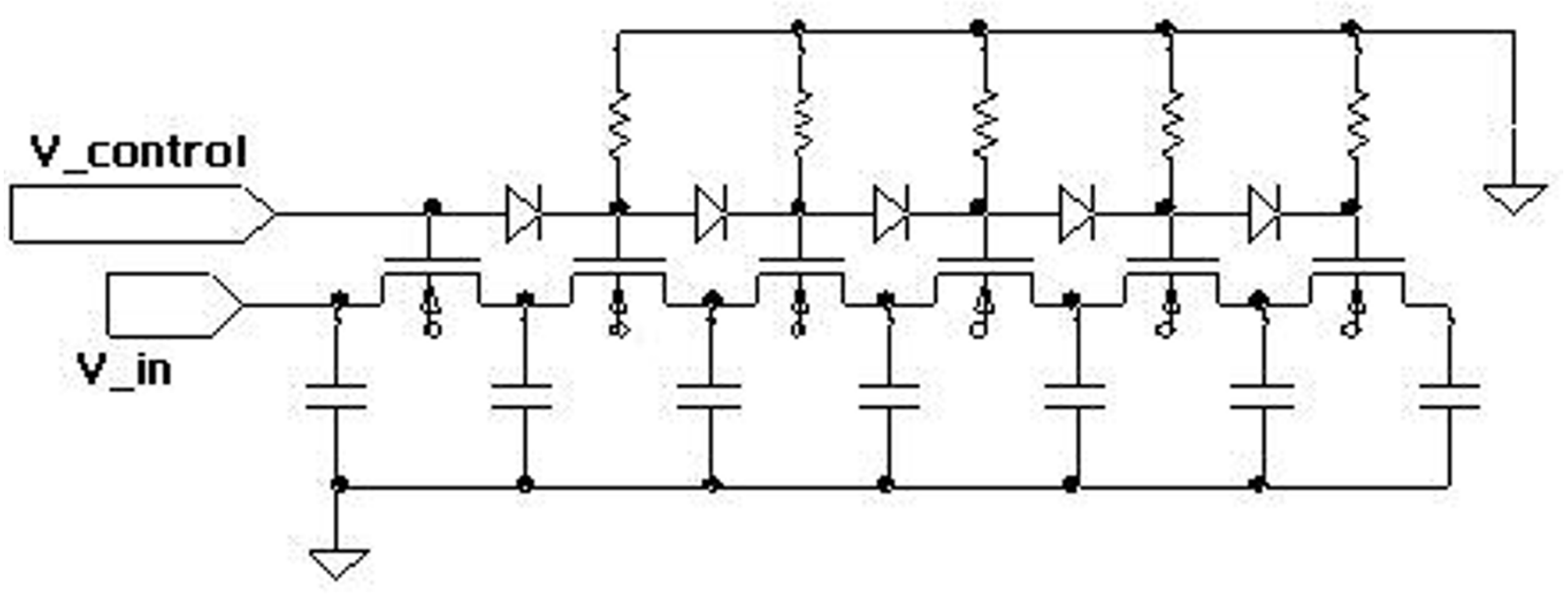

Figure 10

Capacitive bank using diodes and MOSFETs

$$
R=\frac{9 \eta}{2 \rho(a+b t)^{2}}
$$

where, $t$ refers to pregnancy time (expressed as periods of 8 weeks) and the vessel radius is assumed to vary as $(a+$ $b t)$.

In normal pregnancy, vessel radius varies from $0.14 \mathrm{~cm}$ to $0.20 \mathrm{~cm}$ in 25 weeks. Thus, $b$ is small. Therefore,

$(a+b t)^{2} \approx a^{2}+2 a b t=A+B t$

Substituting the above expression in equation. (6), we get

$$
R=\frac{9 \eta}{2 \rho(A+B t)}
$$

Comparing equation (5) and (7), we get an expression for $\mathrm{V}_{\mathrm{GS}}$ in terms of pregnancy time. Thus, the transmission line resistance is directly controlled with a voltage that is in turn proportional to pregnancy time.

\section{Transmission line capacitance}

A passive capacitance is used to model the capacitance in transmission line. Its value is proportional to density of blood and radius of vessel, and inversely proportional to Elasticity of arterial wall and the thickness of arterial wall.

Transmission line inductance

A passive inductance is used to model inductance in transmission line.

\section{Load resistance}

The load resistance is also implemented using an NMOS. Load resistance decreases as pregnancy matures.

\section{Load capacitance}

The variation in transmission line inductance is mapped onto the load capacitance. Load capacitance now varies as, it is implemented using a bank of capacitors, consisting of passive capacitance in parallel, with MOS switches connecting them (Fig. 10). The control voltage decides the gate voltage of MOSFETs. This gate voltage, in turn, decides the number of capacitors that add in parallel in the circuit. Thus, a voltage-controlled capacitance is obtained. Since load capacitance decreases with time, therefore the control voltage for this capacitive bank is decreased with pregnancy time. Also, since the decrease in capacitance is inverse square w.r.t. Radius of the vessel, and the control voltage is taken to be proportional to pregnancy time; therefore, the passive capacitance values decrease inverse-squarely in the bank. 
Matching resistance

Variation in matching resistance is neglected.

\section{Matching capacitance}

Matching capacitance is also implemented using a bank of capacitors. This capacitance varies linearly with pregnancy time.

\section{Matching Impedance $\left(C_{m}, R_{m}\right)$}

The matching impedance is needed at source to absorb the waves that come after reflection from the load.

\section{Authors' contributions}

Author 1(AG) and Author 2 (SKD) constructed the model and carried out the entire simulation and various modifications outlined in the manuscript and prepared the technical-engineering part of the manuscript. Author 3 (ASG) developed and conceived the non-linear mathematical and FET model in place of the known linear TL model, carried out the Doppler image analysis, performed clinical diagnostic Doppler study and provided necessary medical inputs, and finalized the paper. Author 4 (DNC) suggested the MOSFET model over the original JFET model, coordinated the study, and provided the expertise in designing, modifying and problem solving of the MOSFET.

\section{Acknowledgements}

We would like to thank all the anonymous reviewers for their helpful comments.

\section{References}

I. Brosen IA, Robertson WB and Dixon HG: The physiological response of the vessel of the placental bed to normal pregnancy. J Path Bacteriol 1967, 93:569-79.

2. Easterling TR, Beneditti TJ, Schmuker BC and Millard SP: Maternal Haemodynamics in normal and pre-eclamptic pregnancies-a long study. Obstet Gynaecol 1990, 76:1061-1069.

3. Sengupta A and Guha SK: Multifactorial interactions in the Aetiopathogenesis of EPH-gestosis - a hypothesis. Medical Hypothesis 1994, 43:322-326.

4. Sengupta A, Biswas P, Jayaraman G and Guha SK: Understanding the uteroplacental blood flow in normal and abnormal pregnancy through a mathematical model. Med Biol Engineering and Computing 1997, 35:223-230.

5. Adamson SL, Morrow RJ, Bascom PAJ, Mo IYL and Ritchie JWK: Effect of placental resistance, arterial diameter, and blood pressure on the uterine arterial velocity waveform: A computer modeling approach. Ultrasound Med Biol 1989, I 5:437-42.

6. Mo LY, Bascom PAJ, Ritchie $K$ and McCowan ME: A transmission line modelling approach to the interpretation of uterine Doppler Waveforms. Ultrasound Med Biol 1988, I4:365-376.

7. Sengupta A: A biotechnological study of the pathophysiology of the uteroplacental vascular system in pregnancy and its related complications of pre-eclampsia. PhD thesis. Indian Institute of Technology, Delhi. Center for Biomedical Engineering 1995.

8. Sengupta A, Biswas P and Guha SK: Prediction of uterine Doppler velocity waveform using Junction Field Effect Transistor model [Abstract]. In the abstracts of the 29th International OG Congress: Japan 1997.

9. Oosteroff $H$, Wichers G, Fidler $V$ and Aanoudse JG: Blood viscosity and uterine artery flow velocity waveforms in pregnancy. A longitudinal study. Placenta 1993, I4:555-56I.
10. Leifso C, Haslett JW and McRory JG: Monolithic Tunable Active Inductor with Independent $\mathbf{Q}$ Control. IEEE Trans. Microwave Th. and Techniques 2000, 48: 1024-1029.

II. Brown MA, North L and Hargood J: Uteroplacental Doppler Ultrasound in routine antenatal care. Austral NZ J Obs Gyne 1990, 30:303-307.

12. Loguet P: Influence of increase blood pressure \& AGII on uteroplacental \& fetoplacental blood velocity indices in the human. Clinical Science 1990, 78:95-I00.

13. Mahelak KE, Rosenberg J, Berkowitz GS, Chitkara U and Berkowitz KL: Umbilical \& uterine artery flow velocity waveform. Ultrasound Med Biol 1989, 8: I7I-I76.

14. Sengupta A: An experimental study to evaluate the technological limitation in the understanding of the haemodynamic change in pre-eclampsia. Blood Pressure Monitoring 1998, 3:24I-245.

15. Pries AR, Secomb TW, Gaehtgai P and Gross JF: Blood flow in microvascular networks experiment \& simulation. Circulation Research 1990, 67:826-834.

16. Sugihara-seki M, Minamijama $M$ and Hanai S: Vascular research of arterioles with non-uniform diameter. Microvascular Research 1989, 38:148-154.

17. Rabaey JM: Digital Integrated Circuits: A Design Perspective. USA: Prentice Hall; 1996:39-57.

18. Rideout VC and Dick DE: Difference-Differential Equations for Fluid Flow in Distensible Tubes. IEEE Trans. Biomed. Eng 1967, |4:|7|-|77. Publish with Biomed Central and every
scientist can read your work free of charge

"BioMed Central will be the most significant development for disseminating the results of biomedical research in our lifetime. "

Sir Paul Nurse, Cancer Research UK

Your research papers will be:

- available free of charge to the entire biomedical community

- peer reviewed and published immediately upon acceptance

- cited in PubMed and archived on PubMed Central

- yours - you keep the copyright
BioMedcentral 